\title{
Controlled generation of ultra-short electron bunches using density modulation
}

\author{
Samuel R. Yoffe ${ }^{\mathrm{a}}$, Bernhard Ersfeld ${ }^{\mathrm{a}}$, Matthew P. Tooley ${ }^{\mathrm{a}, \mathrm{b}}$, Adam Noble ${ }^{\mathrm{a}}$, Ross Fraser ${ }^{\mathrm{a}}$, and \\ Dino A. Jaroszynski ${ }^{a}$

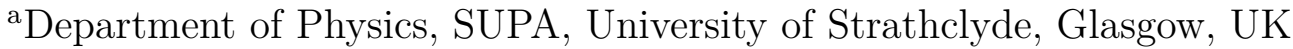 \\ ${ }^{b}$ Research Software Engineering, University of Sheffield, Sheffield, UK
}

\begin{abstract}
Stimulated electron self-injection in the laser wakefield accelerator (LWFA) using density downramps is well known and regularly used to produce high energy electron bunches. The use of density gradients not only to stimulate injection but also control the properties of the injected electron bunch was recently presented by Tooley et al. [Phys. Rev. Lett. 119, 044801 (2017)], in which the authors put forward a model for controlling the velocity of the back of the bubble and compared to 2D and 3D particle-in-cell (PIC) data. This model is discussed and used to identify suitable LWFA parameters for ultra-short injection and repeated injection of multiple bunches. Quasi-3D PIC data is used to demonstrate injection of multiple bunches well separated in energy.
\end{abstract}

Keywords: Laser wakefield accelerator, LWFA, self-injection, ultra-short electron bunches, attosecond bunches

\section{INTODUCTION}

Next generation particle accelerators based on laser-plasma interactions are enabling access to electron beam parameters not previously possible with conventional technologies. Radio frequency (RF) accelerators have bunch durations limited to several 10s of femtoseconds and their gradients are limited to $\sim 100 \mathrm{MV} / \mathrm{m}$. The limited gradients result in $10 \mathrm{~s}$ of metres to kilometres long accelerators to reach $\mathrm{GeV}$ energies. In contrast, plasma waves driven by intense ultra-short duration laser pulses ${ }^{1}$ can produce accelerating fields up to TV/m, which is more than thee orders of magnitude higher than possible using conventional RF cavities. This allows accelerator lengths to be shrunk to millimetres, while simultaneously reducing electron bunch durations ${ }^{2}$ to attoseconds. This favourable down-scaling is resulting in a paradigm shift in accelerator applications, largely because ultrashort bunches are useful as as basis for brilliant sources of incoherent and coherent X-rays, ${ }^{3}$ which have numerous applications.

An intense laser pulse propagating through underdense plasma displaces particles away from the laser axis due to the ponderomotive force. On the timescale of the interaction, the plasma ions remain stationary, with the resulting charge separation producing a strong transverse electric field in which the electrons undergo oscillation at the betatron frequency. This region evacuated of electrons, known as the bubble, follows behind the laser at its group velocity. Figure 1 shows the bubble structure, with its dense electron sheath, and the resulting electric fields.

In addition to the strong transverse focussing field, ${ }^{4}$ the ion cavity of a laser wakefield accelerator (LWFA) produces a strong longitudinal accelerating field, which is also illustrated in Figure 1. Electrons that enter the accelerating field are rapidly accelerated to high energies. ${ }^{5-7}$ A method for capturing these electrons from the plasma itself (rather than external sources) is often referred to as self-injection. Control over when and where electrons can be injected is important for tuning the accelerator for a particular applications. If bunches of accelerated electrons with extremely short duration (attoseconds) could be reliably produced, they would find application in ultra-fast imaging and time resolved spectroscopy using radiation sources such as free-electron lasers $^{8,9}$ (FELs) and synchrotrons.

Further author information: (Send correspondence to D.A.J.)

D.A.J.: E-mail: d.a.jaroszynski@strath.ac.uk

S.R.Y.: E-mail: sam.yoffe@strath.ac.uk 


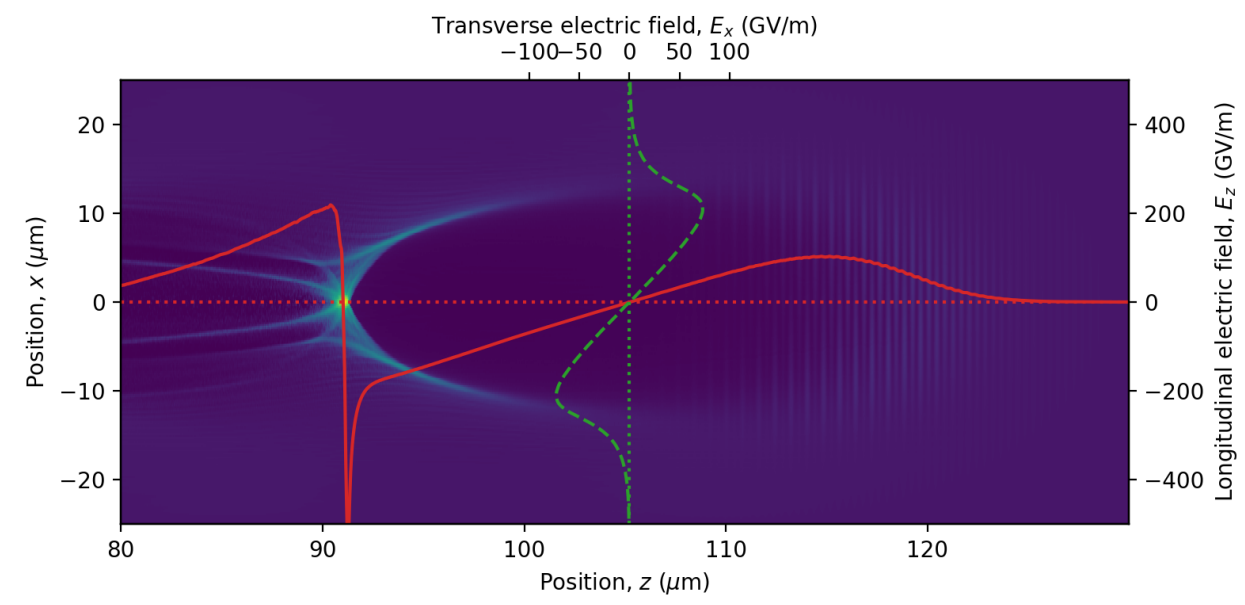

Figure 1: The electron number density is shown, illustrating the bubble and dense electron sheath, for a plasma density $n_{0}=2 \times 10^{18} \mathrm{~cm}^{-3}$ and dimensionless laser amplitude $a_{0}=3$. Electrons injected into the accelerating phase of the LWFA experience a strong longitudinal field of $100 \mathrm{~s} \mathrm{GV} / \mathrm{m}$, and are focussed by a transverse field of around $100 \mathrm{GV} / \mathrm{m}$.

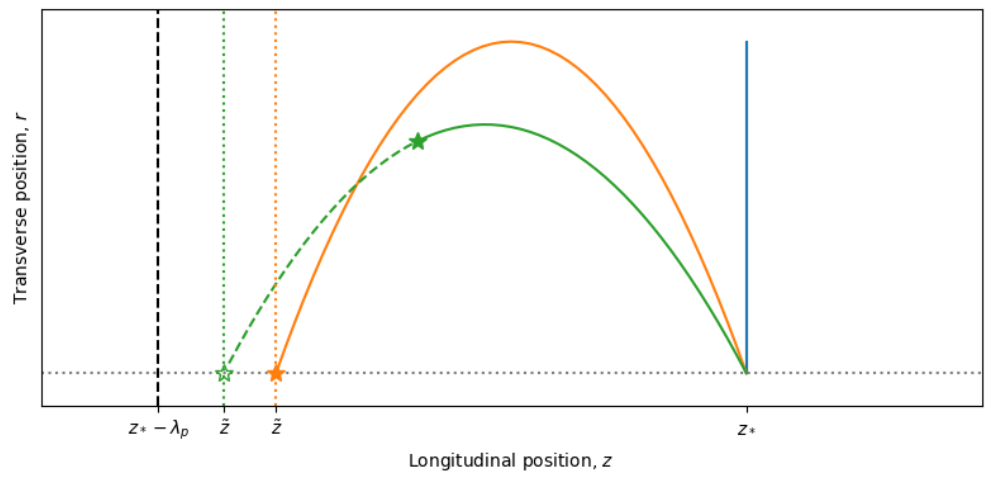

Figure 2: Example electron trajectories forming the back of the bubble at position $z_{*}$ and time $t_{b}$. Electrons with longitudinal momentum and off-axis initial positions can be treated as electrons ejected on axis from positions within a plasma wavelength, $\lambda_{p}$.

\section{MODELLING ELECTRON SELF-INJECTION}

A model for the conditions required for electron self-injection caused by density and laser intensity gradients was presented by Tooley et al. ${ }^{10}$ The model considers the trajectory of an electron displaced from the laser axis that undergoes half a plasma oscillation, where an electron kicked at time $t_{\ell}$ will arrive back on the axis to form the rear of the bubble at time

$$
t_{b}=t_{\ell}+\tau,
$$

where $\tau=\pi / \omega_{\beta}$ and betatron frequency $\omega_{\beta}=\omega_{p} / \sqrt{\Gamma_{e}}$, with the plasma frequency $\omega_{p}=e \sqrt{n / m_{e} \epsilon_{0}}$ and $\Gamma_{e}=\kappa \gamma_{e}$ contains the response to the laser in the electron Lorentz factor, $\gamma_{e}(\kappa=1(2)$ in $2 \mathrm{D}(3 \mathrm{D}))$. The velocity of this position marking the rear of the bubble can then be found by differentiating with respect to position,

$$
\frac{1}{\beta_{b}}=\frac{1}{\beta_{g}}+c \frac{d \tau}{d z}=\frac{1}{\beta_{g}}+\frac{\lambda}{2} \frac{d}{d z}\left(\frac{\sqrt{\Gamma_{e}}}{\eta}\right),
$$

where $\eta^{2}=n / n_{\text {crit }}$ and $n_{\text {crit }}$ is the critical density for the laser wavelength used. 

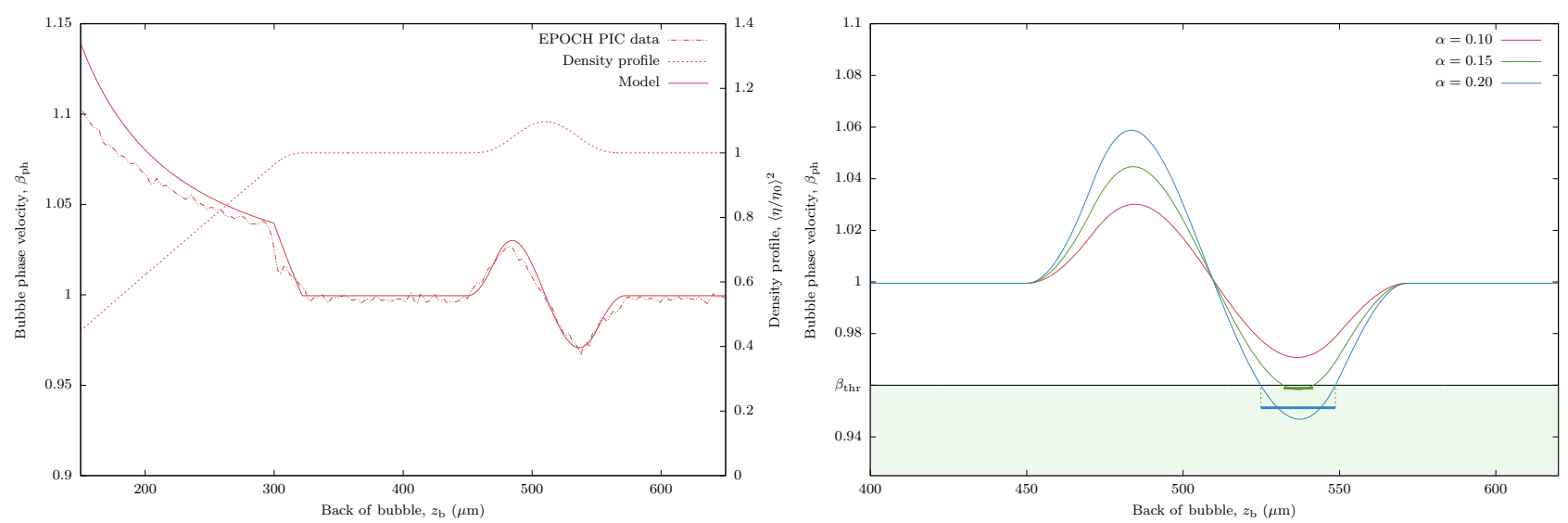

Figure 3: (Left) PIC data showing the density modulation (dashed, right axis) and resulting bubble velocity variation (dot-dashed), along with the bubble velocity predicted by the model (solid). (Right) The variation of the bubble velocity where the plasma density gradient has been varied by controlling the (relative) height of the density modulation (increasing $\alpha$ ). The electron treshold velocity $\beta_{\text {thr }}$ extracted from a non-injecting PIC simulation is plotted, and the region for which stimulated injection can occur is shaded green.

In the LWFA, electrons are not simply displaced transversely from the laser propagation axis and nowhere else: most electrons are displaced from initial positions off-axis, and all gain a longitudinal kick. Both of these effects can be modelled as electrons that cross at the back of the bubble, position $z_{*}$, but whose trajectories originated on-axis at a position $\tilde{z} \leq z_{*}$. Figure 2 shows a purely transverse trajectory starting on-axis in blue, leaving from and returning to $z_{*}$, along with on- and off-axis trajectories (orange and green) with effective on-axis origins at $\tilde{z}$. To capture the effects of these electrons, the equation is averaged over positions within one plasma wavelength, $z-\lambda_{p}<\tilde{z}<z$, resulting in

$$
\beta_{b}=\beta_{g}\left(1+\frac{\lambda \beta_{g}}{2} \frac{d}{d z}\left\langle\frac{\sqrt{\Gamma_{e}}}{\eta}\right\rangle\right)^{-1} .
$$

This equation demonstrates the dependence of the bubble velocity on the electron density gradients and laser intensity variations. An increase to the density leads to a shortening of the bubble, and an associated increase to the velocity of the back of the bubble, whereas a plasma density decrease results in a lengthening of the bubble and a corresponding reduction of the bubble velocity.

For electrons to be injected into the accelerating structure, a necessary condition is that, when they return to the axis, they are travelling faster than the back of the bubble. If this is not satisfied, then electrons cannot enter the bubble and experience the accelerating field. Therefore, plasma density downramps can be used to slow the back of the bubble and thus allow electrons that are not otherwise moving fast enough to be injected to now enter into the bubble.

A comparison of the model to particle-in-cell data for the bubble velocity is given in Figure 3 (left). The density modulation and the calculated bubble velocity are presented, along with the prediction from the model. The agreement is excellent.

The maximum longitudinal velocity of electrons returning to the laser axis can be obtained from a noninjecting PIC simulation, and is referred to as the threshold velocity, $\beta_{\mathrm{thr}}$. When the bubble velocity drops below this value, $\beta_{b}<\beta_{\text {thr }}$, we expect electrons to be captured by the bubble and accelerated. This threshold is shown in Figure 3 (right). The positions for which this criterion is satisfied can be used to predict the injected electron bunch length, and estimate the charge, as shown in Figure 4. As can be seen, the agreement is excellent.

\section{MULTIPLE ELECTRON BUNCH INJECTION}

The controlled injection of multiple short-duration electron bunches has many applications, such as drivers for free-electron lasers (FELs) or synchrotron sources. To investigate stimulated injection of multiple bunches 


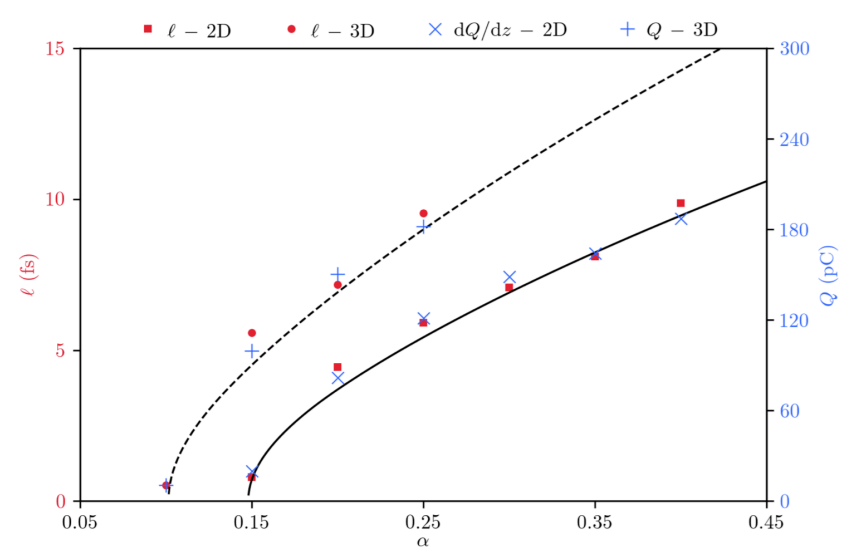

Figure 4: Comparison of predicted electron bunch length and estimated charge and values measured from PIC data for $2 \mathrm{D}$ and $3 \mathrm{D}$ cases.
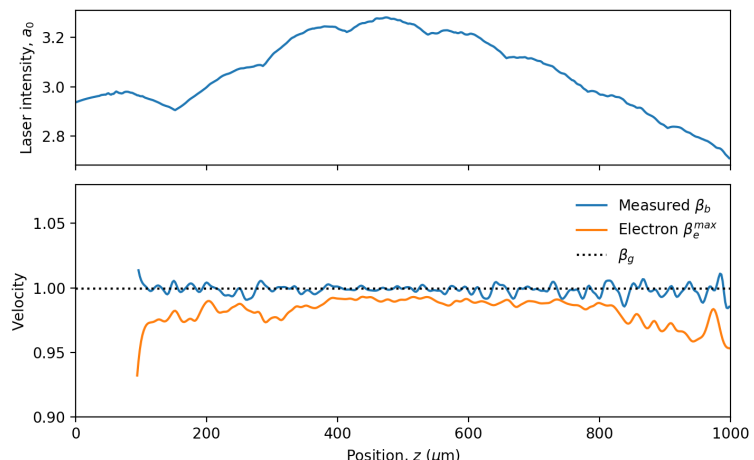

(a)

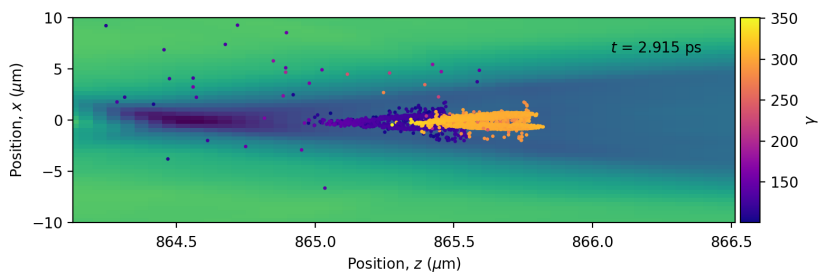

(c)
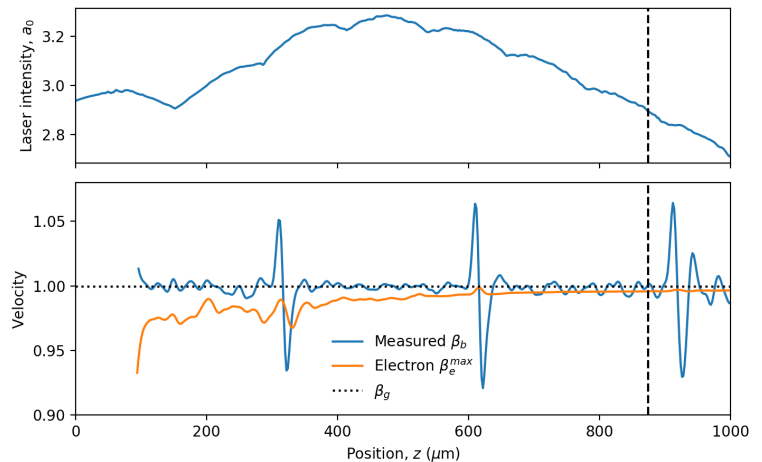

(b)

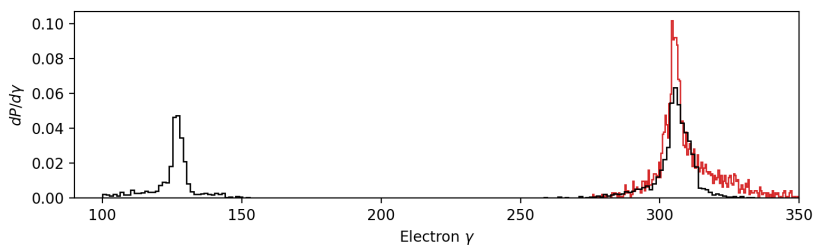

(d)

Figure 5: Part (a): Evolution of laser amplitude, and bubble and electron velocity for a non-injecting simulation using parameters given in the text. Part (b): Evolution with multiple controlled injections using density modulations separated by $300 \mu \mathrm{m}$. Part (c): Snapshot at the position indicated by the dashed line in part (b), showing two spatially-overlapping electron bunches separated in energy. Part (d): Comparison of the energy spectrum for stimulated single-bunch (red) and dual injection (black).

controlled by plasma density modulations, we need to ensure that the variation of the laser intensity caused by self-focussing and diffraction do not stimulate spontaneous injection. Figure 5(a) shows the variation of laser amplitude, bubble velocity, and maximum electron velocity for a non-injecting case, using a $30 \mathrm{fs} 800 \mathrm{~nm}$ laser with initial dimensionless amplitude $a_{0}=3$ and spot size $12 \mu \mathrm{m}$ with an electron density $n_{0}=2 \times 10^{18} \mathrm{~cm}^{-3}$. While the laser amplitude is observed to vary in time, the resulting changes to the bubble velocity are not large enough to stimulate injection. 
In Figure 5(b), multiple cosine-squared density modulations separated by $300 \mu \mathrm{m}$ have been imposed. As the laser passes over these modulations, the bubble velocity is observed to drop below the maximum electron velocity, suggesting that injection can take place. Part (c) shows a snapshot of the electric field towards the back of the bubble at time $t=2.915 \mathrm{ps}$, corresponding to the position indicated in part (b) by the black dashed line. Therefore, we expect to observe two injected electron bunches, as illustrated. The electrons are coloured by their Lorentz factor, $\gamma$, showing that there are two injection events that result in a pair of spatially-overlapping electron bunches, which are well separated in energy. The latter statement is confirmed by the energy spectra shown in part (d), which compares the spectrum for a single-injection simulation with that for the double injection case. While overlapping in space, the difference in energy between the bunches (caused by different times spent in the accelerator) means that the bunches could be separated for a particular application.

There are some changes to the properties of the leading electron bunch caused by the injection of the second into the LWFA. For example, with a single density modulation the injected electron bunch has a root-meansquare duration of 374 as and contains $24.32 \mathrm{pC}$ charge, whereas in the case of double injection, the leading bunch is reduced slightly to $24.25 \mathrm{pC}$ contained in (r.m.s.) $370 \mathrm{as}$, while the second bunch contains $13.86 \mathrm{pC}$ and has a duration of 398 as. We note that multiple controlled injection of short bunches offers a mechanism to increase total injected charge while maintaining short bunch durations.

\section{CONCLUSION}

The injection of short-duration electron bunches controlled by density modulations has been modelled and verified using 2D and 3D PIC data, with excellent agreement. Controlled repeated injection of multiple electron bunches has been demonstrated using parameters for which the laser evolution does not cause spontaneous electron injection. The injected electron bunches, while spatially overlapping, have well-defined, distinct energies which would enable separation if needed. Multiple injection allows the total injected charge to be increased while maintaining ultrashort, low energy spread electron bunches. Additional injection is observed to alter the properties of the initial electron bunch; in this case, the first bunch is determined to be slightly shorter and have a slightly higher peak current. Further stimulated injections could be performed, however the consequences of beam loading may begin to play a role in the injection process, since the fields will be modified by the presence of the injected bunches within the bubble.

\section{ACKNOWLEDGMENTS}

This work was supported by the UK EPSRC grant number EP/N028694/1. The authors are grateful to the developers of the open-source FBPIC particle-in-cell code (github.com/fbpic/fbpic). ${ }^{11}$ Data were also obtained using the EPOCH PIC code, ${ }^{12}$ in part funded by the UK EPSRC grants EP/G054950/1, EP/G056803/1, EP/G055165/1 and EP/ M022463/1. This work used the ARCHER UK National Supercomputing Service (http://www.archer.ac.uk), the ARCHIE-WeSt High Performance Computer (www.archie-west.ac.uk), and GPU resources on the Cambridge Service for Data-Driven Discovery (CSD3, https://www.hpc.cam.ac.uk). Data are openly available online from the University of Strathclyde KnowledgeBase. ${ }^{13,14}$

\section{REFERENCES}

[1] Tajima, T. and Dawson, J. M., "Laser electron accelerator," Phys. Rev. Lett. 43, 267-270 (Jul 1979).

[2] Islam, M. R., Brunetti, E., Shanks, R. P., Ersfeld, B., Issac, R. C., Cipiccia, S., Anania, M. P., Welsh, G. H., Wiggins, S. M., Noble, A., Cairns, R. A., Raj, G., and Jaroszynski, D. A., "Near-threshold electron injection in the laser-plasma wakefield accelerator leading to femtosecond bunches," New Journal of Physics 17, 093033 (sep 2015).

[3] Jaroszynski, D. A., Bingham, R., Brunetti, E., Ersfeld, B., Gallacher, J., van der Geer, B., Issac, R., Jamison, S. P., Jones, D., de Loos, M., Lyachev, A., Pavlov, V., Reitsma, A., Saveliev, Y., Vieux, G., and Wiggins, S. M., "Radiation sources based on laser-plasma interactions," Philosophical Transactions of the Royal Society A: Mathematical, Physical and Engineering Sciences 364, 689-710 (2006). 
[4] Cipiccia, S., Islam, M. R., Ersfeld, B., Shanks, R. P., Brunetti, E., Vieux, G., Yang, X., Issac, R. C., Wiggins, S. M., Welsh, G. H., Anania, M.-P., Maneuski, D., Montgomery, R., Smith, G., Hoek, M., Hamilton, D. J., Lemos, N. R. C., Symes, D., Rajeev, P. P., Shea, V. O., ao M. Dias, J., and Jaroszynski, D. A., "Gamma-rays from harmonically resonant betatron oscillations in a plasma wake," Nature Physics 7, 867-871 (2011).

[5] Mangles, S. P. D., Murphy, C. D., Najmudin, Z., Thomas, A. G. R., Collier, J. L., Dangor, A. E., Divall, E. J., Foster, P. S., Gallacher, J. G., Hooker, C. J., Jaroszynski, D. A., Langley, A. J., Mori, W. B., Norreys, P. A., Tsung, F. S., Viskup, R., Walton, B. R., and Krushelnick, K., "Monoenergetic beams of relativistic electrons from intense laser-plasma interactions," Nature 431, 535-538 (2004).

[6] Geddes, C. G. R., Toth, C., van Tilborg, J., Esarey, E., Schroeder, C. B., Bruhwiler, D., Nieter, C., Cary, J., and Leemans, W. P., "High-quality electron beams from a laser wakefield accelerator using plasma-channel guiding," Nature 431, 538-541 (2004).

[7] Faure, J., Glinec, Y., Pukhov, A., Kiselev, S., Gordienko, S., Lefebvre, E., Rousseau, J.-P., Burgy, F., and Malka, V., "A laser-plasma accelerator producing monoenergetic electron beams," Nature 431, 541-544 (2004).

[8] Jaroszynski, D. A. and Vieux, G., "Coherent radiation sources based on laser plasma accelerators," $10^{\text {th }}$ Workshop on Advanced Accelerator Concepts, 902-913 (2002).

[9] Schlenvoigt, H.-P., Haupt, K., Debus, A., Budde, F., Jäckel, O., Pfotenhauer, S., Schwoerer, H., Rohwer, E., Gallacher, J. G., Brunetti, E., Shanks, R. P., Wiggins, S. M., and Jaroszynski, D. A., "A compact synchrotron radiation source driven by a laser-plasma wakefield accelerator," Nature Physics 4, 130-133 (2008).

[10] Tooley, M. P., Ersfeld, B., Yoffe, S. R., Noble, A., Brunetti, E., Sheng, Z. M., Islam, M. R., and Jaroszynski, D. A., "Towards attosecond high-energy electron bunches: Controlling self-injection in laser-wakefield accelerators through plasma-density modulation," Phys. Rev. Lett. 119, 044801 (2017).

[11] Lehe, R., Kirchen, M., Andriyash, I. A., Godfrey, B. B., and Vay, J.-L., "A spectral, quasi-cylindrical and dispersion-free particle-in-cell algorithm," Computer Physics Communications 203, 66 - 82 (2016).

[12] Arber, T. D., Bennett, K., Brady, C. S., Lawrence-Douglas, A., Ramsay, M. G., Sircombe, N. J., Gillies, P., Evans, R. G., Schmitz, H., Bell, A. R., and Ridgers, C. P., "Contemporary particle-in-cell approach to laser-plasma modelling," Plasma Physics and Controlled Fusion 57(11), 113001 (2015).

[13] See https://doi.org/10.15129/4b58d502-415f-4064-b3b7-5966dbc0e679.

[14] See http://dx.doi.org/10.15129/f9a49809-f797-4ea7-8f65-ed39ce14da18. 